\title{
RENDIMIENTO ESCOLAR EN AlumNOS DE NIVEL SUPERIOR DEL IPN
}

José Gonzalo Amador Salinas, Virginia González Rivera, Juan Pablo Balderrama Camacho, Paulina Cerecedo Vázquez, Jeanette del Carmen López Orendain, Ingrid Raquel Rivera Pérez y Brenda Viridiana Suárez Gutiérrez

\section{INSTITUTO POLITÉCNICO NACIONAL CENTRO INTERDISCIPLINARIO DE CIENCIAS DE LA SALUD - UNIDAD SANTO TOMÁS}

\section{RESUMEN}

En el Instituto Politécnico Nacional existe una evidente preocupación por la deserción y bajo desempeño académico debido a que los alumnos permanecen mayor tiempo en la escuela utilizando los recursos por más tiempo del programado y presupuestado, lo que implica un costo extra para la institución. Dada esta situación, en el presente trabajo se observa si existen diferencias en el rendimiento escolar de alumnos de nivel superior del CICS UST en relación a 5 variables socioeducativas: licenciatura, turno escolar, sexo, nivel escolar de la madre y horas de sueño. Se aplicaron 2 cuestionarios, el primero para establecer el perfil socio-económicoacadémico de los alumnos de Odontología, Optometría y Psicología; posteriormente se les aplicó el segundo cuestionario Ilamado Índice de Calidad de Sueño de Pittsburg. De acuerdo con los resultados que se encontraron hay diferencias significativas en cuanto al rendimiento escolar en las tres licenciaturas, dependiendo del nivel escolar de la madre y en cuanto a las horas del sueño.

Palabras Clave: Patrones de sueño, horas de sueño, calidad del sueño, rendimiento académico, nivel académico.

\section{SCHOOL PERFORMANCE IN STUDENTS OF UNIVERSITY EDUCATION FROM IPN}

\begin{abstract}
In the National Polytechnic Institute there is an obvious concern regarding the desertion and low academic performance because this makes the students spend a longer time in school using the resources for a longer period than originally scheduled and budgeted, which implies an extra cost for the institution. Given this situation on the present work is observed if there are differences in the academic performance of upper level students from the CICS UST in relationship with 5 socio educational variables: bachelor degree, school shift, gender, mother school level and sleep hours. 2 questionnaires were applied, the first one in order to stablish the socio-economic-academic profile of the students of Odontology, optometry and psychology, afterwards was applied the second questionnaire named Pittsburgh Sleep Quality Index. According to the results found, there are significant differences regarding the academic performance of the three bachelor degrees, depending on the mother's school level and sleep hours.
\end{abstract}

Keywords: Sleeps patterns, sleep time, sleep quality, academic performance, academic level. 
BitÁCORA DEL ARTí́culo

\section{RENDIMIENTO ESCOLAR EN ALUMNOS DE NIVEL SUPERIOR DEL IPN}

José Gonzalo Amador Salinas, Virginia González Rivera, Juan Pablo Balderrama Camacho, Paulina Cerecedo Vázquez, Jeanette del Carmen López Orendain, Ingrid Raquel Rivera Pérez y Brenda Viridiana Suárez Gutiérrez

\section{Bitácora del Artículo:}

Recibido: 9 de septiembre de 2014

Aceptado: 5 de febrero de 2015

Publicado en línea: 7 de febrero de 2015

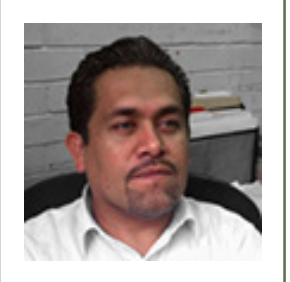

José Gonzalo Amador Salinas Instituto Politécnico Nacional

Correo: g-zama@hotmail.com

Profesor de tiempo completo Titular A, jefe delDepartamento de Innovación Educativa en el Centro Interdisciplinario de Ciencias de la Salud Unidad Santo Tomás del IPN.

Ver más..

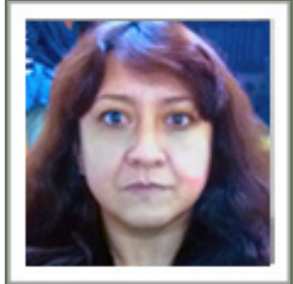

Virginia González Rivera Instituto Politécnico Nacional

Correo: vicky_gori@live.com.mx

Profesora Titular A, de la licenciatura en Psicología en el Centro Interdisciplinario de Ciencias de la Salud Unidad Santo Tomás del IPN.

Ver más...

\section{Contribución de los autores/as}

José Gonzalo Amador Salinas y Virginia González Rivera en la revisión bibliográfica, construcción del método, discusión y en la redacción general del artículo | Juan Pablo Balderrama Camacho, Paulina Cerecedo Vázquez, Jeanette del Carmen López Orendain, Ingrid Raquel Rivera Pérez y Brenda Viridiana Suárez Gutiérrez participaron en el trabajo de campo recolectando datos, búsqueda bibliográfica, captura de datos.

\section{Agradecimientos}

Agradacemos al Centro Interdisciplinario de Ciencias de la Salud, Unidad Santo Tomás del Instituto Polítecnico Nacional por las facilidades prestadas para la realización de este estudio.

\section{Datos de filiación de los autores}

José Gonzalo Amador Salinas | Carrera de Psicología, Centro Interdisciplinario de Ciencias de la Salud, Unidad Santo Tomás del Instituto Politécnico Nacional IPN | Virginia González Rivera | Carrera de Psicología, Centro Interdisciplinario de Ciencias de la Salud, Unidad Santo Tomás del Instituto Politécnico Nacional IPN | 


\section{TABLA DE CONTENIDO}

INTRODUCCIÓN

MÉTODO

95

Sujetos, 95

Escenario, 95

Aparatos y materiales, 95

Instrumento, 95

RESULTADOS

96

Discusión

97

CONCLUSIONES

99

REFERENCIAS

100

Meta-AnÁlisis del Artículo 102

Dimensión Cuantitativa, 103

Dimensión Cualitativa, 105

IR A LA Historia del Proceso Editorial

112 
Introducción

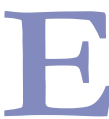
n México, sólo el $12 \%$ de las personas entre 55 y 64 años de edad han obtenido una educación universitaria, mientras que el $23 \%$ de los jóvenes de 25 a 34 años no han tenido esta educación. Aun cuando este porcentaje de egresados universitarios sigue siendo muy inferior a la media de la OCDE (39\%), los niveles de educación superior entre los jóvenes han aumentado 6 puntos porcentuales entre el año 2000 (17\%) y el 2011 (23\%); ya superan a los de Austria (21\%), Brasil (13\%), Italia (21\%) y Turquía (19\%) (OCDE, 2013).

Hablando específicamente del Instituto Politécnico Nacional, al rendir el informe 2010-2012, la directora general del IPN, Yoloxóchitl Bustamante Díez, afirmó que en el nivel superior los estudiantes aprobados se incrementaron más de 10 por ciento respecto al ciclo 2008-2009; los desertores disminuyeron 52 por ciento al retener 5 mil 782 alumnos más, y la eficiencia terminal alcanzó 61.9 por ciento (IPN, 2013). Generalmente, existe preocupación por la deserción y bajo desempeño académico porque los alumnos permanecen mayor tiempo en la escuela utilizando los recursos por más tiempo del programado y presupuestado, lo que implica un costo extra para la institución.

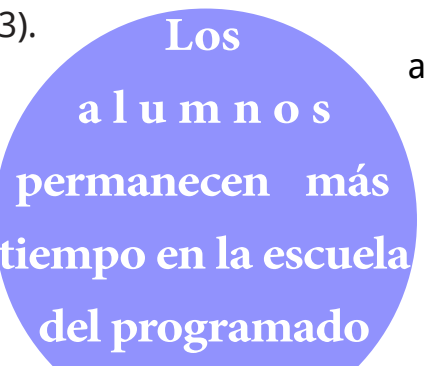

De tal manera que existen dos problemas académicos importantes en los alumnos del nivel superior del IPN, uno son los índices de deserción y el otro es el bajo rendimiento académico. Este último es el que compete a nuestra investigación y podría ser medido de diferentes maneras, por ejemplo, con el número de materias aprobadas, el resultado de una prueba en específico (exámenes del CENEVAL), o el promedio de calificaciones, siendo ésta una de las líneas más investigadas, principalmente desde la perspectiva de factores relacionados con el mismo estudiante (endógenos) y los propios del mundo circundante (exógenos) (Porcel, E., Dapozo, G. y López, M., 2010).

Precisamente hay opiniones que apuntan a diversos planteamientos de la investigación educativa, contemplando como variable dependiente el rendimiento académico y aproximadamente 228 variables independientes, las cuales podemos clasificar en 6 constructos a saber según Wang (1993; citado por Martínez R., en Castañeda, 2004):
1. Características de la organización y gobierno del sistema educativo, en el nivel estatal y de distrito. Variables demográficas del distrito escolar y variables políticas.

2. Características del contexto social y familiar. Variables relativas a las características de la comunidad, pares, el uso del tiempo extraescolar y ambiente del hogar; por ejemplo, apoyo de los padres.

3. Características de la escuela. Demografía escolar, toma de decisiones, cultura de la escuela, organización y políticas, accesibilidad (por ejemplo: asistencia a clases), participación de los padres en las actividades de la escuela.

4. Currículo. Característica demográficas del programa (por ejemplo: tamaño de los grupos), congruencia entre currículo y enseñanza, apoyos (por ejemplo becas).

5. Prácticas de enseñanza. Acciones de apoyo, métodos de enseñanza, cantidad de instrucción, evaluación, administración del aula, interacción entre maestro y alumno de tipo social, interacción académica, clima del aula.

6. Características de los alumnos. Género y edad, antecedentes escolares, características sociales y conductuales, características motivacionales y afectivas, características cognitivas, meta cognitivas y psicomotrices.

Dicho lo anterior realizaremos una breve revisión de diferentes autores que han estudiado distintas de estas variables con relación al desempeño escolar. Alcover (2007, citado en Porcel y cols. 2010), han realizado investigaciones que concluyeron que el rendimiento escolar de un alumno se puede predecir en función de las variables socioeducativas como el promedio previo (promedio en escuela anterior) de tal manera que el rendimiento universitario puede ser explicado a partir del rendimiento en el nivel de educación medio o secundario (Ponsot, Varela, Sinha y Valera, 2009; citado en Porcel y cols. 2010).

Por otro lado, en su investigación Dapozo, G. y López, M. (2010) concluyen que el desempeño en la preparación previa a la universidad es positiva si la orientación del currículo del bachillerato está orientada al área de los estudios universitarios; por ejemplo, en el IPN, en el nivel medio superior, se imparten programas 
académicos de ciencias físico matemáticas, medico biológicas, sociales y administrativas, que permiten a los alumnos relacionar lo que ahí aprenden con lo que pueden aprender en el nivel superior, lo cual debería traducirse en recomendaciones a los alumnos sobre estos datos y sobre todo en la orientación vocacional.

Otra variable importante a tomar en cuenta en el rendimiento escolar son los medios para seleccionar a los alumnos en su ingreso a las Instituciones de Educación Superior (IES) con la aplicación de exámenes de selección, el cual a su vez, es un importante factor de predicción del rendimiento escolar en su ingreso a una IES. Algunos autores argumentan que las pruebas de ingreso pueden usarse de manera predictiva (Martínez, R. et al., 2000; en Chain, 2003). Estos estudios se realizan mediante enfoques que buscan demostrar estadísticamente el grado de asociación entre variables, por ejemplo la calificación obtenida en el examen y el promedio final de calificaciones en la licenciatura (Chain, 2003).

Por otro lado, en un estudio realizado por Baltra, M. (2010) sugiere que en promedio los alumnos pertenecientes a niveles socioeconómicos bajos les es difícil alcanzar niveles superiores de desempeño escolar; en cambio los alumnos con un estrato socioeconómico medio bajo, muestran niveles de desempeño constante; en contraste, un porcentaje alto de alumnos de estrato socioeconómico medio muestran un nivel de desempeño que aumenta en forma exponencial.

De acuerdo con el autor, esto muestra que la formación de las distintas habilidades cognitivas (síntesis, análisis y solución de problemas) se ve afectada por el nivel socioeconómico de los estudiantes y que existen diferencias entre los estratos socioeconómicos y el desempeño escolar.

Con respecto a las características del alumno, ha habido un vacío en cuanto a investigación se refiere, por ejemplo, una variable no muy investigada es la relación entre las horas que duermen los alumnos y el rendimiento escolar. Dement y Guilleminault (1973; citado en Botella C., en Méndez y Macia, 1998), mencionan que existen 3 criterios para calificar los problemas en la conducta de dormir:

1. Conciliación del sueño (tardar en conciliar el sueño más de 30 minutos).

2. Interrupción del dormir (los periodos en los que permanece despierto deben exceder los 30 minutos).
3. Total de horas del dormir (debe ser menor a seis horas y media).

Bootzin y Nicasio (1978; citado en Macia, 1998) mencionan que el estrés y los hábitos mal establecidos se traducirán en problemas con la conducta de dormir (por ejemplo: leer en la cama, idear, buscar soluciones, etc.). De esta manera, presentan un estudio de caso en el que una adolescente muestra problemas con la conducta inicial de dormir, ya que tarda hasta más de 2 horas en conciliar el sueño, lo anterior hace que se muestre cansada y que su rendimiento académico sea menor. El tratamiento consistió en la higiene del sueño y control del estímulo.

En otro estudio, en el que examinan el sueño y calidad de vida, Miró, Cano y Buela (2005) establecen que los patrones de sueño son: corto, cuando se duerme menos de 6 horas por noche; medio, cuando se duerme entre 6 y 9 horas por noche, y largo, cuando se duerme más de 9 horas por noche. Un cuarto tipo serían los sujetos con patrón de sueño variable que se caracterizarían por la inconsistencia de sus hábitos de sueño (Quevedo B. V., 2011). Por otro lado, en el estudio pionero de Kripke, Simons, Garfinkel y Hammond (1979, recién reevaluado por Kripke, Garfinkel, Wingard, Klauber y Marter; citados por Miró y cols., 2005) encontraron que el mínimo de mortalidad ocurrió en las personas que dormían habitualmente entre siete y ocho horas (patrón de sueño medio), de la misma manera, los sujetos con patrón de sueño intermedio obtienen las mejores puntuaciones de salud física o psicológica; además, las quejas de somnolencia suelen manifestarse con mayor frecuencia tanto en los patrones de sueño cortos como en los largos, lo que se ha asociado a bajo funcionamiento intelectual, pobre calidad de vida, tasa de accidentabilidad aumentada, mayor riesgo de consumir café, alcohol o drogas y bajo rendimiento académico.

Algunos estudios que comparan la duración habitual del sueño de los jóvenes de 1963 con los de 1910-1911 (Carskadon, 1993; citado en Ángeles, Miróy Buela, 2003), y de 1974 hasta el 2003 (Iglowstein, Jenni, Molinari y Largo, 2003) encuentran una reducción del tiempo total de sueño de aproximadamente una hora y media. Los efectos negativos de la reducción parcial del sueño sobre una amplia gama de respuestas fisiológicas, cognitivas, conductuales y emocionales han sido demostradas por Belenky, Wesensten y Thome (2003; Dinges, Pack, Williams y cols., 1997; citados en Ángeles I., Miró, Catena y Buela, 2003); sin embargo, no se han realizado los estudios necesarios para establecer la relación entre duración del sueño y el rendimiento escolar. 
Dada la falta de información que hay sobre rendimiento escolar y su relación con algunas variables, planteamos como objetivo del presente trabajo: observar si existen diferencias en el rendimiento escolar de alumnos de nivel superior del CICS UST en relación a 5 variables socioeducativas: licenciatura (Odontología, Optometría y Psicología), turno escolar (matutino o vespertino), sexo (masculino o femenino), nivel escolar de la madre (primaria y secundaria, preparatoria, licenciatura, posgrado) y horas de sueño (patrón de sueño corto, patrón de sueño medio y patrón de sueño largo).

\section{Método}

\section{Sujetos}

Se realizó un muestreo por conveniencia para la aplicación de 300 encuestas a 101 estudiantes de Psicología, 100 estudiantes de Odontología y 99 alumnos de Optometría del CICS UST. 172 alumnos pertenecían al turno matutino y 128 eran vespertinos. De los cuales 171 eran femeninos y 129 masculinos. Respecto al semestre, 74 alumnos cursaban el primer semestre, 75 cursaban el tercer semestre, 76 el quinto semestre y 75 estaban en séptimo semestre. Finalmente, la edad de los alumnos encuestados fue: 31 reportaron tener 17 años, 53 dijeron tener 18 años, 58 mencionaron tener 19 años, mientras que 63 dijeron tener 20 años, 67 reportaron contar con 21 años, sólo 15 dijeron tener 22 años, mientras que 4 mencionaron tener 23 años, 5 dijeron tener 24 años y sólo 4 reportaron 25 años. El porcentaje de alumnos encuestados fue de aproximadamente el $12 \%$ de la población total del CICS UST.

\section{Escenario}

La aplicación se llevó a cabo en la sala del Centro de Ayuda y Prevención Psicológica CAPPSI con horarios programados para cada grupo de las tres licenciaturas: Odontología, Optometría y Psicología.

\section{Aparatos y materiales}

Hojas, lápices, plumas, computadoras, plataforma virtual desarrollada con el software Moodle, disponible en: http://www.virtual.cics-sto.ipn.mx/UTyCV/moodle/, paquete estadístico SPSS, versión 20.

\section{Instrumento}

Cuestionario de perfil socioeconómico que consta de una sección de datos generales, valores, planificación del estudio, técnicas de aprendizaje, ingresos familiares, tipo de equipo de cómputo usado, etc., y el cuestionario Índice de Calidad de Sueño de Pittsburg (ICSP), el cual proporciona una puntuación global de la calidad en siete componentes distintos: calidad subjetiva del sueño, latencia (cantidad de tiempo que lleva conciliar el sueño), duración, eficiencia habitual, alteraciones, uso de medicación hipnótica y disfunción diurna. El cuestionario ICSP muestra una consistencia interna (coeficiente a de Cronbach de 0.83), una sensibilidad del $89.6 \%$ y una especificidad del $86.5 \%$ (Buysse et al., 1989; Buysse, Reynolds y Monk, 1991; Royuela y Macias, 1997; citados en Quevedo B. V. y Quevedo B.R., 2011).

\section{Procedimiento}

El estudio fue de tipo no experimental, transversal, bajo un paradigma epistemológico/metodológico cuantitativo debido a que se aplicaron 2 cuestionarios con variables definidas en escala de intervalo. Se aplicó el primer cuestionario a 855 alumnos a través de la plataforma virtual del CICS-UST, disponible en: http:// www.virtual.cics-sto.ipn.mx/UTyCV/moodle/.

En los resultados de dicha encuesta se observaron diferencias en las estrategias de estudio y diferencias en las horas de sueño entre los estudiantes de las tres carreras (ver Tabla 1), con base en una muestra de conveniencia se procedió a aplicar el cuestionario de Índice de Calidad de Sueño de Pittsburg, a 300 estudiantes sin diagnóstico de trastorno del sueño (el número de alumnos se determinó con base en la fórmula del tamaño de la muestra). Cabe mencionar que en los cuestionarios aplicados se excluyó el apartado del nombre para proteger la identidad de los alumnos; se programó a los grupos de las tres licenciaturas, y la aplicación del mismo estuvo a cargo de los alumnos de Psicología de la unidad de aprendizaje "Proyectos de Investigación Cuantitativa"; ellos explicaron el propósito del estudio y manifestaron que toda la información recolectada sería resguardada por el departamento de Innovación Educativa y usada con fines académicos y de investigación. Al aplicar el cuestionario, se procedió a darles las instrucciones y se descartaron a los sujetos que reportaran tener diagnóstico de un trastorno del sueño, 5 cuestionarios fueron invalidados por estar incompletos; posteriormente se llevó a cabo el vaciado de los datos del cuestionario en el programa estadístico SPSS, en el cual se realizaron análisis de varianza ANOVA y comparación de medias con el estadístico t de Student para muestras independientes.

En esta investigación nuestra variable dependiente es el rendimiento académico que se define como 
Tabla 1.

Perfil socio-económico-académico de los alumnos del CICS-UST (Encuesta 1)

\begin{tabular}{|c|c|c|c|c|c|c|c|c|c|}
\hline Licenciatura & Edad & Género & $\begin{array}{l}\text { Estado } \\
\text { civil }\end{array}$ & $\begin{array}{l}\text { Valores con menor } \\
\text { puntuación }\end{array}$ & $\begin{array}{l}\text { Estrategias de estudio con } \\
\text { menor puntuación }\end{array}$ & $\begin{array}{l}\text { Horas de } \\
\text { estudio }\end{array}$ & $\begin{array}{l}\text { Tiempo de } \\
\text { recorrido }\end{array}$ & $\begin{array}{l}\text { Horas de } \\
\text { sueño }\end{array}$ & $\begin{array}{l}\text { Nivel de } \\
\text { alerta }\end{array}$ \\
\hline Optometría & $\begin{array}{l}73 \% \\
\text { tiene } \\
\text { entre } 17 \\
\text { y } 20\end{array}$ & $\begin{array}{l}77 \% \text { son de } \\
\text { género } \\
\text { femenino }\end{array}$ & $\begin{array}{l}\text { El } 97 \% \\
\text { son } \\
\text { solteros }\end{array}$ & $\begin{array}{l}\text { Responsabilidad, } \\
\text { tolerancia, disciplina y } \\
\text { discreción }\end{array}$ & Planificación del estudio & $\begin{array}{l}34 \% \\
\text { estudia de } \\
1 \text { a } 3 \text { horas }\end{array}$ & $\begin{array}{l}\text { El } 33 \% \text { hace de } \\
\text { recorrido más de } \\
1 \text { hora y media }\end{array}$ & $\begin{array}{l}\text { El } 66 \% \text { dijo } \\
\text { dormir de } 3 \\
\text { a } 5 \text { horas }\end{array}$ & $\begin{array}{l}33 \% \text { dijo } \\
\text { sentirse } \\
\text { cansados }\end{array}$ \\
\hline Odontología & $\begin{array}{l}75 \% \\
\text { tiene } \\
\text { entre } 17 \\
\text { y } 20\end{array}$ & $\begin{array}{l}67 \% \text { son de } \\
\text { género } \\
\text { femenino }\end{array}$ & $\begin{array}{l}\text { El } 95 \% \\
\text { son } \\
\text { solteros }\end{array}$ & $\begin{array}{l}\text { Discreción, disciplina y } \\
\text { tolerancia }\end{array}$ & $\begin{array}{l}\text { Planificación del estudio, } \\
\text { elaboración de esquemas, buscar } \\
\text { palabras desconocidas, la selección } \\
\text { de ideas principales, participación } \\
\text { en clase }\end{array}$ & $\begin{array}{l}34 \% \\
\text { estudia de } \\
4 \text { a } 6 \text { horas }\end{array}$ & $\begin{array}{l}\text { El } 33 \% \text { hace de } \\
\text { recorrido } 1 \text { hora }\end{array}$ & $\begin{array}{l}\text { El } 50 \% \text { dice } \\
\text { dormir de } 6 \\
\text { a } 9 \text { horas }\end{array}$ & $\begin{array}{l}41 \% \text { dice } \\
\text { sentirse } \\
\text { cansado }\end{array}$ \\
\hline Psicología & $\begin{array}{l}60 \% \\
\text { tiene } \\
\text { entre } 17 \\
\text { y } 20\end{array}$ & $\begin{array}{l}73 \% \text { son de } \\
\text { género } \\
\text { femenino }\end{array}$ & $\begin{array}{l}\text { El } 97 \% \\
\text { son } \\
\text { solteros }\end{array}$ & Disciplina y tolerancia & $\begin{array}{l}\text { Elaboración de esquemas, organizar } \\
\text { apuntes, preguntar al profesor, ir a } \\
\text { la biblioteca y destacar las ideas } \\
\text { más relevantes }\end{array}$ & $\begin{array}{l}29 \% \\
\text { estudia de } \\
1 \text { a } 3 \text { horas }\end{array}$ & $\begin{array}{l}\text { El } 35 \% \text { hace de } \\
\text { recorrido más de } \\
1 \text { hora }\end{array}$ & $\begin{array}{l}\text { El } 60 \% \text { dijo } \\
\text { dormir de } 3 \\
\text { a } 5 \text { horas }\end{array}$ & $\begin{array}{l}42 \% \text { dijo } \\
\text { sentirse } \\
\text { cansados }\end{array}$ \\
\hline
\end{tabular}

el promedio general que los alumnos tienen en la licenciatura. Las variables independientes son identificadas como: licenciatura a la que pertenecen los alumnos, turno escolar, sexo, nivel escolar de la madre y horas de sueño.

Dicho lo anterior nuestra hipótesis es que existen diferencias significativas en el rendimiento escolar de alumnos de nivel superior del CICS UST en relación a 5 variables socioeducativas (licenciatura, turno escolar, sexo, nivel escolar de la madre y horas de sueño).

\section{Resultados}

De acuerdo con los datos obtenidos encontramos que el promedio de calificaciones reportado por los alumnos encuestados fue de 8.0007 (Tabla 2), mientras que el promedio de calificaciones por licenciatura fue para Psicología: 8.3030; Odontología: 7.8609, y Optometría: 7.8336 (Tabla 3).

De esta manera para comparar las diferencias entre las licenciaturas con el rendimiento académico, se aplicó la prueba estadística ANOVA, con la cual se verificó que existen diferencias estadísticamente significativas Tabla 2.

Promedio del desempeño escolar de los alumnos encuestados.

\begin{tabular}{|c|c|c|}
\hline \multicolumn{3}{|c|}{ Estadísticos descriptivos } \\
\hline$n$ & Media \\
\hline Promedio & 300 & 8.0007 \\
\hline Válido & 300 & \\
\hline
\end{tabular}

en función de los promedios de las 3 licenciaturas ( $g l=2 ; F=9.52 ; p=0.000)$, al realizar las comparaciones múltiples, se encuentra que existen diferencias estadísticamente significativas entre los promedios de Psicología con Odontología y Optometría, y no hay diferencias significativas entre los promedios de Odontología y Optometría.

Tabla 3 .

$$
\begin{aligned}
& \text { Comparación de promedios por } \\
& \text { licenciatura. }
\end{aligned}
$$

\begin{tabular}{|c|c|c|}
\hline \multicolumn{3}{|c|}{ Promedio académico } \\
\hline Licenciatura & Media & $\mathrm{N}$ \\
\hline Psicología & 8.3030 & 101 \\
\hline Optometría & 7.8336 & 100 \\
\hline Odontología & 7.8609 & 99 \\
\hline Total & 8.0007 & 300 \\
\hline
\end{tabular}

También se comparó el turno en el que están inscritos los participantes y el rendimiento académico, siendo el promedio de los alumnos del turno matutino 7.98 y de los alumnos del turno vespertino 8.02, al usar la prueba t de Student para muestras independientes, se encuentra que las diferencias entre ambos turnos no son estadísticamente significativas ( $\mathrm{gl}=298 ; \mathrm{F}=2.129$; $\mathrm{p}=0.706$ ). Así mismo, se comparó la relación entre el sexo y el promedio, siendo la media de las mujeres 8.0380 y de los hombres 7.9512 , resultando las diferencias no significativas ( $g \mathrm{l}=298 ; \mathrm{F}=1.077 ; \mathrm{p}=0.399$ ).

Por otro lado, se comparó el nivel de estudios de la madre y el promedio de calificaciones (el promedio 
de los sujetos con madres con estudios de primaria o secundaria fue de 7.8026, preparatoria 7.9923, licenciatura 8.1666, posgrado 8.6142) por medio de la prueba estadística ANOVA, existiendo diferencias significativas entre el nivel de estudios de primaria/ secundaria y posgrado $(g l=3 ; F=3.511 ; p=0.016)$ y no hay diferencias significativas entre los demás niveles de estudio de la madre.

Otro análisis realizado fue la comparación con los patrones de sueño y el promedio, a través de la prueba estadística ANOVA, siendo que los alumnos que reportaron tener patrones de sueño corto, obtuvieron en promedio 8.1, mientras que los de sueño medio 7.8 y los de sueño largo 7.7, al comparar las diferencias entre los patrones de sueño, se obtuvo que hay diferencias estadísticamente significativas ya que: ( $g l=2 ; F=3.454 ; p=0.033$ ).

Tabla 4.

Promedio de horas de sueño por licenciatura

\begin{tabular}{|c|c|c|c|}
\hline Licenciatura & Media & $n$ & Desv. Típ. \\
\hline Psicología & 4.42 & 101 & 2.013 \\
\hline Optometría & 5.53 & 100 & 1.592 \\
\hline Odontología & 5.03 & 99 & 2.445 \\
\hline Total & 4.99 & 300 & 2.089 \\
\hline
\end{tabular}

Finalmente para comparar las diferencias entre licenciaturas respecto a las horas de sueño, se aplicó la ANOVA, con la cual se verificó que existen diferencias estadísticamente significativas en función de las horas de sueño de las 3 licenciaturas ( $g l=2 ; F=7.454 ; p=0.001$ ) (Tabla 4). Al realizar las comparaciones múltiples, se encuentra que existen diferencias estadísticamente significativas entre las horas de sueño reportadas por los alumnos de Optometría y Psicología $(p<0.05)$ y no existieron diferencias estadísticamente significativas entre las horas de sueño reportadas por los alumnos de las licenciaturas de Optometría y Odontología $(p>0.05)$.

Tabla 5.

Comparación del promedio de horas de sueño por sexo

\begin{tabular}{|l|c|c|}
\hline \multicolumn{1}{|c|}{ Sexo } & Media & N \\
\hline Femenino & 5,01 & 171 \\
\hline Masculino & 4,96 & 129 \\
\hline Total & 4,99 & 300 \\
\hline
\end{tabular}

Al comparar mediante la prueba $t$ de Student para muestras independientes el promedio de horas
Tabla 6 .

Promedio de horas de sueño por turno

\begin{tabular}{|l|c|c|c|}
\hline Turno & Media & $\mathrm{n}$ & Desv. típ. \\
\hline Matutino & 4.97 & 172 & 2.150 \\
\hline Vespertino & 5.02 & 128 & 2.012 \\
\hline Total & 4.99 & 300 & 2.089 \\
\hline
\end{tabular}

de sueño por sexo, se encontró que la media de las mujeres fue de 5,01 horas y de los hombres de 4,96 horas; sin embargo, las diferencias no fueron estadísticamente significativas $(\mathrm{gl}=298 ; \quad \mathrm{F}=.494$; $\mathrm{p}=0.822$ ) (Tabla 5); por último, al comparar las horas de sueño por turno se encontró que no hay diferencias estadísticamente significativas ya que se presentó el siguiente valor:(gl=298; $F=.020 ; p=0.861$ ) (Tabla 6).

\section{Discusión}

El primer punto abordado en este trabajo fue el perfil socio-económico-académico de los alumnos del CICSUST que se realizó por medio del cuestionario 1, los resultados de la encuesta apoyan los reportados por diversos autores (Elías T. B., 2007; Wang, 1993; citados por Martínez, en Castañeda, 2004) acerca de que las técnicas de aprendizaje más usadas por los alumnos de licenciatura son tomar apuntes en clases, hacer resúmenes y repasar los apuntes, por el contrario son pocos los que piden asesorías a los docentes. En nuestro caso, las técnicas de aprendizaje menos usadas son preguntar al profesor, la planificación del estudio, la elaboración de esquemas, buscar palabras desconocidas, la selección de ideas principales, participación en clase, organizar apuntes, ir a la biblioteca y destacar las ideas más relevantes (ver Tabla1).

Otro punto a destacar en ese cuestionario es el alto porcentaje de alumnos que reportan dormir patrones de sueño corto.

Con respecto a la primera variable socioeducativa analizada (licenciatura), encontramos que el promedio general de los 300 encuestados fue de 8.0007, en Psicología fue de 8.3030, en Optometría de 7.8336, y en Odontología 7.8609; tras realizar la comparación de varianzas, se concluye que existen diferencias significativas entre la licenciatura en Psicología con Odontología y Optometría $(p<0.05)$, entre Odontología y Optometría no hay diferencias significativas ya que el valor $(p>0.05)$, lo anterior puede ser explicado por as prácticas de enseñanza (métodos de enseñanza, 
cantidad de instrucción, evaluación, administración del aula, interacción entre maestro y alumno de tipo social, interacción académica, clima del aula), variables que pertenecen al punto 5 según Wang (1993; citado por Martínez, en Castañeda, 2004), las cuales podrían ser muy diferentes en las licenciaturas estudiadas.

Por otro lado, al preguntar ¿cuántas horas de sueño real has mantenido por las noches?, los alumnos del turno matutino en promedio respondieron que 4.97 horas, y los del turno vespertino 5.02 horas, esto es similar a lo reportado por estudios como los de Chol, Jinkwan, Sangduck, Yongkyu y Soonjae (2003), quienes en una muestra de 3871 estudiantes adolescentes, reportan que el número de horas promedio que duermen los sujetos entrevistados es de 6.4 horas por día, lo que representa apenas un patrón de sueño medio; también los estudios de Moura, Soares, Parente, Moura, Luciana y Coelho Damasceno (2013), quienes en una muestra de 701 estudiantes universitarios, encontraron un promedio de sueño de 6.3 horas de sueño. En nuestro estudio, las menores horas de sueño pueden estar relacionadas con las largas distancias que recorren los alumnos. Volviendo a nuestros datos, al comparar por licenciatura las horas de sueño, se encontró que los alumnos de Psicología reportaron dormir en promedio 4.42 horas, en Optometría 5.53 horas y en Odontología 5.03 horas; hay diferencias significativas ya que los alumnos de Psicología reportaron dormir menos que los de las licenciaturas en Odontología y Optometría, siendo también éstos los que reportan un mejor promedio.

Al comparar los patrones de sueño con el rendimiento académico, nuestros resultados con adultos jóvenes son diferentes a los de Kelman (1999; en Quevedo y Quevedo 2011; Ladellas, Chamarro, Badia, Oberst y Carbonell, 2011) ya que según sus datos, el rendimiento académico en niños y adolescentes se ve afectado cuando hay falta de sueño, favoreciendo que los sujetos que poseen un patrón de sueño medio obtengan significativamente mejores calificaciones en comparación con los sujetos que presentan un patrón de sueño corto y largo (las notas que obtienen son muy parecidas), la explicación de lo anterior sería mostrado por el estudio de Touchette, Petit, Séguin, Boivin y Tremblay (2007) acerca de que los adolescentes por su etapa de desarrollo necesitan dormir un patrón de sueño medio, pero por distintas exigencias sociales, presentan un cierto retraso fisiológico del inicio del sueño (tienden a acostarse más tarde de lo habitual y a despertar por la mañana más temprano), siendo más sensibles a la disminución de horas de sueño, por lo que los adultos jóvenes ya estarían acostumbrados a menos horas de sueño y serían menos sensibles a la disminución de horas de sueño.

Por el contrario, nuestros resultados concuerdan con el estudio realizado por Pérez, Talero y González (2006), quienes en una muestra de 318 estudiantes de medicina concluyen que no hay relación entre más horas de sueño o la privación de éste con el rendimiento académico de los estudiantes; (sin embargo, no aclaran qué patrón de sueño duermen sus sujetos), coincidimos además de que es importante revisar las horas de estudio independiente como causa del mejor rendimiento. También coincidimos con los estudios de Eliasson, Eliasson, King, Gould y Eliasson (2002), quienes concluyen que no hay una relación significativa entre el tiempo total de sueño -periodos largos de sueño- y el rendimiento académico de los sujetos adolescentes. Una posible explicación de lo anterior sería lo mencionado por Talero, Durán y Pérez (2013), quienes exponen que debido al proceso de maduración de la infancia a la adultez se instala un patrón de sueño adulto, caracterizado por menor duración total de horas de sueño, ciclos de sueño más largos y menor duración del sueño diurno, lo que lleva a los jóvenes a adaptarse al entorno académico, modificando los hábitos de sueño aprendidos y practicados en casa (Claros, Rodríguez, Forerom, Camargo y Niño, 2013). Además, estudios como los de Al-Hazzaa, Musaiger, Abahussain, Al-Sobayel, Qahwaji y Care (2014) sugieren que los estilos de vida adquiridos en la familia, como la actividad física, los comportamientos sedentarios y hábitos alimentarios están relacionados con la corta duración del sueño (menos de 8 horas al día) entre los adolescentes.

De esta manera, nosotros podemos afirmar que los sujetos con patrones de sueño corto son más frecuentes (Wheaton y Perry, 2011) y a la vez obtienen mejores calificaciones que los sujetos con patrones de sueño medio y largo. Sin embargo, es de destacar que la frecuencia de sujetos con patrones de sueño largo fue significativamente menos frecuente, lo que está de acuerdo con el estudio de Iglowstein, Jenni, Molinari y Largo (2003) acerca de la reducción de horas de sueño a través de las décadas.

Otras variables que se analizaron fueron las referentes al género y el promedio; el promedio de las mujeres fue de 8.0380 y el de los hombres de 7.9512 , al comparar las medias se encontró que no hay diferencias significativas por lo que nuestros resultados apoyan los reportados por autores como Costa y Tabernero (2012) respecto a que la variable género no muestra diferencias significativas con respecto al rendimiento 
académico en adultos jóvenes.

Uno de los factores que se comprobó en nuestro estudio fue el planteado por Alcover (2007; citado en Porcel y cols., 2010) referente a la ocupación de los padres del punto 2, características del contexto social y familiar según Wang (1993; citado por Martínez, en Castañeda, 2004) ya que al comparar entre el nivel de estudios de la madre (primaria y secundaria, preparatoria, licenciatura, posgrado) y el promedio de los alumnos, el valor $p<0.05$ fue significativo comparando el nivel básico y posgrado a favor de este último.

En nuestro trabajo se observa que a medida que los padres tienen mayor nivel de escolaridad, aumenta la posibilidad de que el alumno obtenga buen rendimiento académico. La educación de los padres es considerada, en general, un factor importante para Porcel y cols. (2010) al explicar el rendimiento estudiantil. Podría inferirse que a mayor cantidad de años de educación de los padres, mayor sería la calidad del apoyo al estudio de los hijos.

Finalmente, algunas de las recomendaciones podrían ser las de incentivar en los alumnos el uso de diferentes técnicas de aprendizaje, sobre todo las referidas a las de planificación e identificación de ideas principales, y tener una mayor participación en clases, así como preguntar sus dudas a los profesores. Con respecto al sueño, se debería promover la educación de la higiene del sueño con la finalidad de que se conozca cómo mejorar su calidad y optimizar así los niveles de rendimiento (Griffin, Guerin, Sharry y Drumm, 2010; Ladellas, Chamarro Badia, Oberst y Carbonell, 2011), ya que diversos autores como Gao, Lv, Li, Zhou, Jin, Dang y Li (2014) y Martínez, Pilar, Miró, Sánchez, Díaz, Cáliz, Vlaeyen, Buela y Casal (2014) han señalado que el uso amplio de educación en higiene del sueño, terapia musical, terapia de control de estímulos y el entrenamiento en relajación muscular progresiva tiene efectos positivos en la mejora de la calidad del sueño en estudiantes universitarios.

\section{Conclusiones}

A partir de este trabajo podemos concluir que el tipo de licenciatura influye en el rendimiento escolar y esto puede estar relacionado con los métodos de enseñanza, cantidad de instrucción, evaluación, administración del aula, interacción entre maestro y alumno de tipo social, interacción académica y clima del aula. Con respecto al nivel escolar de la madre concluimos que es una variable que influye en el rendimiento académico, ya que es más factible que un estudiante reciba apoyo y orientación de mejor calidad cuando su madre ha tenido estudios superiores, al contrario de los alumnos que reportaron que sus madres han cursado estudios básicos.

También se encontró que el número de horas de sueño (clasificadas según patrones de sueño corto, medio o largo) influye en el aprovechamiento académico, al parecer si se sigue un patrón de sueño corto el rendimiento académico es mayor en comparación con los patrones de sueño medio y largo; sin embargo, falta investigar si dormir patrones de sueño corto puede tener efectos en áreas cognitivas, conductuales y emocionales. A su vez encontramos que entre las licenciaturas hay diferencias en las horas de sueño, coincidiendo que los alumnos de la licenciatura en Psicología fueron los que reportaron dormir menos horas y al mismo tiempo reportan un mayor rendimiento escolar, lo cual es motivo de futuras investigaciones.

Algunas de las limitaciones de este trabajo tienen que ver con el tipo de muestreo usado; en futuros trabajos, deben considerarse los muestreos probabilísticos; otra limitación tiene que ver con el número de sujetos, por ello es necesario incrementar el número de aplicaciones ya que en nuestro caso fue de 300 sujetos que asciende al $12 \%$ de la población del CICS UST; también resulta indispensable que las aplicaciones puedan llevarse a cabo en distintos momentos de la vida académica, ya que si son aplicados en periodos de evaluación puede afectar el reporte de los participantes; es importante también proponer trabajos en los que se conformen grupos de alumnos con los diferentes patrones de sueño y se verifique por medio de registros las horas que duermen, para que posteriormente realizar pruebas de rendimiento académico.

Para finalizar, sería interesante que en futuras investigaciones los alumnos de primer ingreso participaran en un taller de higiene del sueño y fueran comparados con un grupo control, para que de manera longitudinal se pudiera observar su desempeño académico y su estado en áreas como la cognitiva, conductual y emocional. 


\section{Referencias}

Al-Hazzaa, H. M., Musaiger, A. O., Abahussain, N. A., Al Sobayel, H. I., Qahwaji, D. M. (2014). Lifestyle correlates of self reported sleep duration among Saudi adolescents: A multicentre school based cross sectional study. Health and Development, 40(4), 533-542.

Ángeles I., Miró C. y Buela C. (2003). Calidad de sueño en sujetos con diferentes patrones habituales de sueño. Psicología y salud. 13(2). Recuperado de http://www.uv.mx/psicysalud/Psicysalud\%2013_2/ index.html

Baltra, M. (2010). Perfiles de desempeño en Matemática, según habilidad cognitiva por nivel socioeconómico en estudiantes chilenos de enseñanza municipal. Revista Electrónica de Investigación Educativa, 12(1). Recuperado de http://redie.uabc.mx/ vol12no1/contenido-baltra.html

Castañeda, F. S. (2004). Educación, aprendizaje y cognición. Teoría en la práctica. México: Manual Moderno.

Chain, R., Cruz, R. N., Martínez, M. M. y Jácome, N. (2003). Examen de selección y probabilidades de éxito escolar en estudios superiores. Estudio en una universidad pública estatal mexicana. Revista Electrónica de Investigación Educativa, 5(1). Recuperado de http://redie.uabc.mx/vol5no1/ contenido-chain.html

Claros, G., Rodríguez, V., Forerom, D., Camargo, S. y Niño, C. (2013). Influencia familiar en el hábito del sueño en una muestra de estudiantes universitarios sanos en Bogotá. Revista Cuidarte, 4(1).

Chol, S., Jinkwan, K., Sangduck, L., Yongkyu, A. y Soonjae, J. (2003). Sleep habits, excessive daytime sleepiness and school performance in high school students. Psychiatry and Clinical Neurosciences, 57(4).

Costa, S. y Tabernero, C. (2012). Rendimiento académico y autoconcepto en estudiantes de Educación Secundaria Obligatoria según el género. Revista Iberoamericana de Psicología y Salud, 3(2).

Eliasson, A., Eliasson, A., King, J., Gould, B., Eliasson, A. (2002). Association of sleep and academic performance. Sleep Breath, 6(1), 45-8.

Faubel, C. (2008). El impacto de la duración habitual del sueño sobre la obesidad, la presión arterial, la calidad de vida y la función cognitiva de los ancianos españoles (Tesis doctoral). Facultad de Medicina, Universidad Autónoma de Madrid.

Gao, R., Lv, Y., Li, X., Zhou, K., Jin, X., Dang, S. y Li, N. (2014). Effects of comprehensive sleep management on sleep quality in university students in mainland china. Sleep and Biological Rhythms, 12(3), 194202.
Griffin, C., Guerin, S., Sharry, J. y Drumm, M. (2010). A multicentre controlled study of an early intervention parenting programme for young children with behavioral and developmental difficulties. International Journal of Clinical and Health Psychology, 10(2).

Iglowstein, I., Jenni, O.G., Molinari, L. y Largo, R.H. (2003). Sleep duration from infancy to adolescence: reference values and generational trends. Pediatrics, 111(2). Recuperado de http://www.ncbi. nlm.nih.gov/pubmed/12563055

Indicadores de la OCDE. (2013). Panorama de la Educación en México. Recuperado de http://www.oecd.org/ edu/Mexico_EAG2013\%20Country\%20note\%20 (ESP).pdf

IPN. (2013). Informe del trienio 2010-2012. Gaceta Politécnica, 15, 3-5. (Número extraordinario 1003)

Ladellas, R., Chamarro, A., Badia, M., Oberst, U. y Carbonell, X. (2011). Efectos de las horas y los hábitos de sueño en el rendimiento académico de niños de 6 y 7 años: un estudio preliminar. Fundación Infancia y Aprendizaje. 23(1), 119-128.

Macia, M. F. (1998). Modificación de conducta con niños y adolescentes. Libro de casos. Madrid: Pirámide.

Martínez, M. Pilar., Miró, Elena., Sánchez, Ana I., Díaz-P., Cáliz R., Vlaeyen, W. S., Buela, C. G. (2014). Cognitivebehavioral therapy for insomnia and sleep hygiene in fibromyalgia: A randomized controlled trial. Journal of Behavioral Medicine, 37(4).

Miró, C. y Buela, C. (2005). Sueño y calidad de vida. Revista Colombiana de Psicología, 14(1). Recuperado de http://dialnet.unirioja.es/servlet/ articulo?codigo $=32458$

Moura, A., Soares, L., Parente, G., Luciana, C. F., Coelho, D. (2013). Evaluación de la calidad del sueño de estudiantes universitarios de Fortaleza-CE. Texto \& Contexto-Enfermagem, 22(2).

OCDE. (2013). Panorama de la educación en México. Recuperado de http://www.oecd.org/edu/Mexico EAG2013\%20Country\%20note\%20(ESP).pdf

Pérez, O. I., Talero, G. y González, R. (2006). Ritmos circadianos de sueño y rendimiento académico en estudiantes de medicina. Revista Ciencia Salud, 4. Bogotá.

Porcel, E., Dapozo, G. y López, M. (2010). Predicción del rendimiento académico de alumnos de primer año de la FACENA (UNNE) en función de su caracterización socioeducativa. Revista Electrónica de Investigación Educativa, 12(2). Recuperado de http://redie.uabc.mx/vol12no2/contenido-porcel dapozo.html

Quevedo, B. V. y Quevedo, B. R. (2011). Influencia del grado de somnolencia, cantidad y calidad de sueño sobre el rendimiento académico en adolescentes. International 
Journal of Clinical and Health Psychology, 11(1).

Rodríguez, F. J. (2010). MEXICANAL. Recuperado de http:// www.mexicanal.com/blog-entry/mtro-franciscogutierrez/29353

Talero, C., Durán, T. y Pérez, I. (2013). Sueño: características generales. Patrones fisiológicos y fisiopatológicos en la adolescencia. Revista Ciencias de la Salud, 11(3). Recuperado de http://www.scielo.org.co/scielo.php?pid=S169272732013000300008\&script=sci_arttext

Touchette, E., Petit, D., Séguin, J., Boivin, M., Tremblay, R. (2007). Associations Between Sleep Duration Patterns and Behavioral/Cognitive Functioning at School Entry. Journal sleep. 30(9). Recuperado de http://www.ncbi.nlm.nih.gov/pmc/articles/ PMC1978413/

Wheaton, Y. y Perry, C. (2011). Effect of Short Sleep Duration on Daily Activities. Morbidity and Mortality Weekly Report. United States, 2005 2008, 60(8), 239-42.

Copyright: @ 2015 Amador, S. J.G., González, R. V., Balderrama, C. J. P., Cerecedo, V. P., López, O. J. C.; Rivera, P. I. R. y Suárez, G. V. B. Este es un artículo de acceso abierto distribuido bajo los términos de la licencia Creative Commons Reconocimiento-NoComercial 4.0 Internacional, por lo que su contenido gráfico y escrito se puede compartir, copiar y redistribuir total o parcialmente sin necesidad de permiso expreso de sus autores/as con la única condición de que no se puede usar con fines directamente comerciales y los términos legales de cualquier trabajo derivado deben ser los mismos que se expresan en la presente declaración. La única condición es que se cite la fuente con referencia a la Revista Digital Internaciona de Psicología y Ciencia Social y a sus autores/es. 


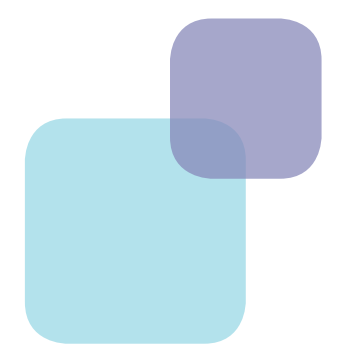

\section{MetA-ANÁlisis del ARTículo}

A continuación se muestran los análisis de las puntuaciones otorgadas a este artículo por sus revisores 


\section{Dimensión Cuantitativa}

\section{Perfil de Evaluación entre Pares}
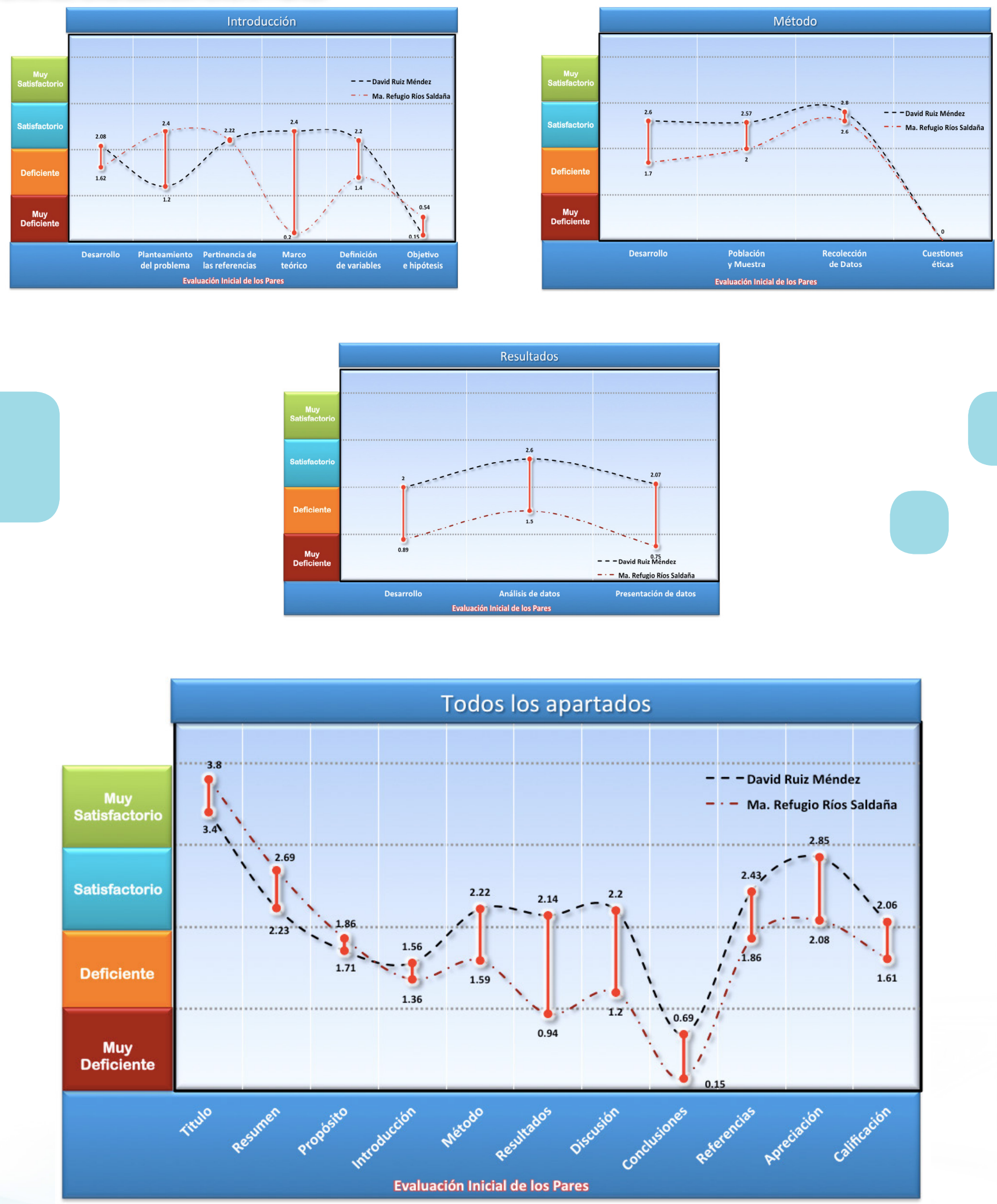


\section{Índice de Concordancia}

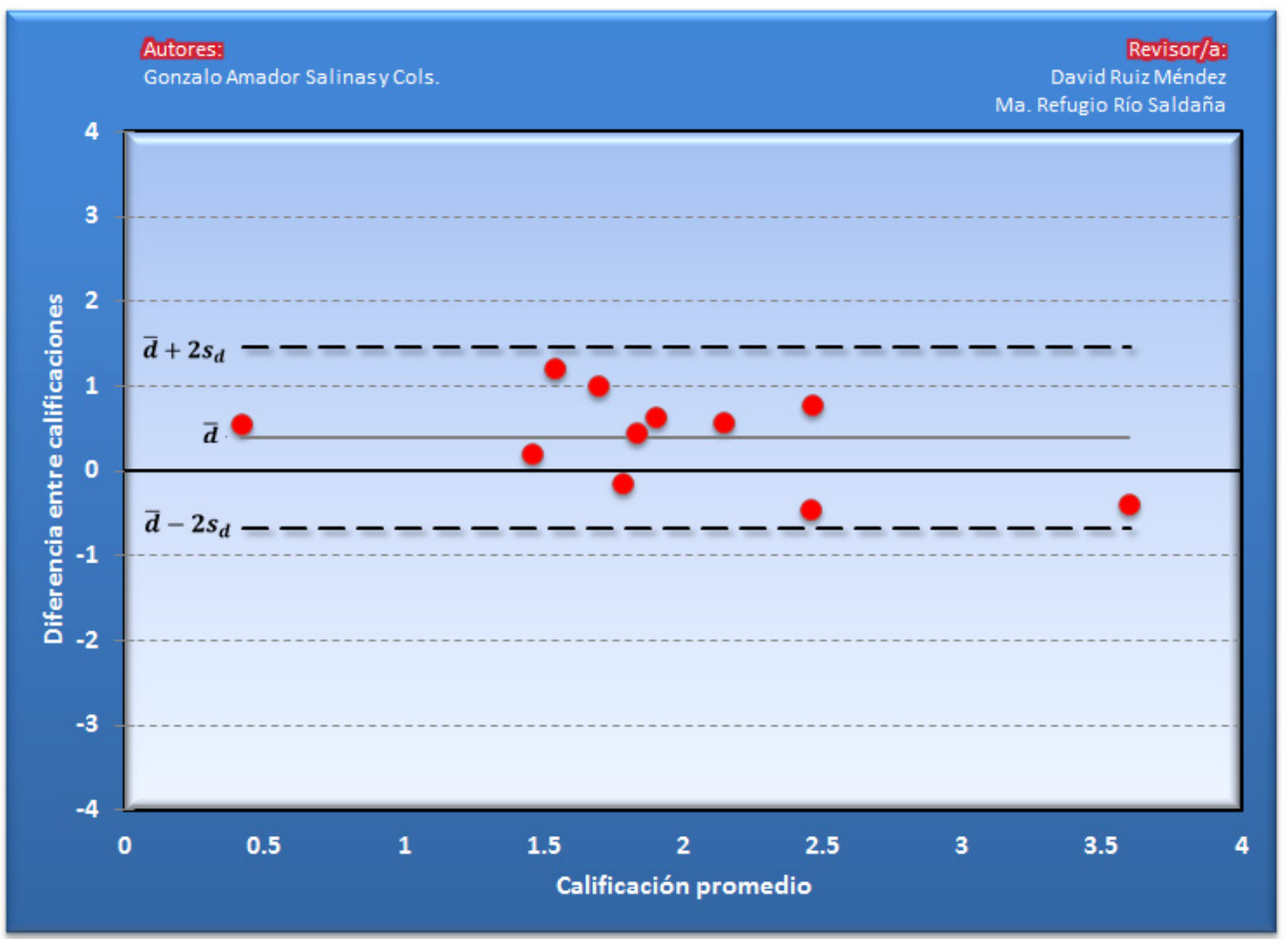

\section{Índice de Acuerdo}

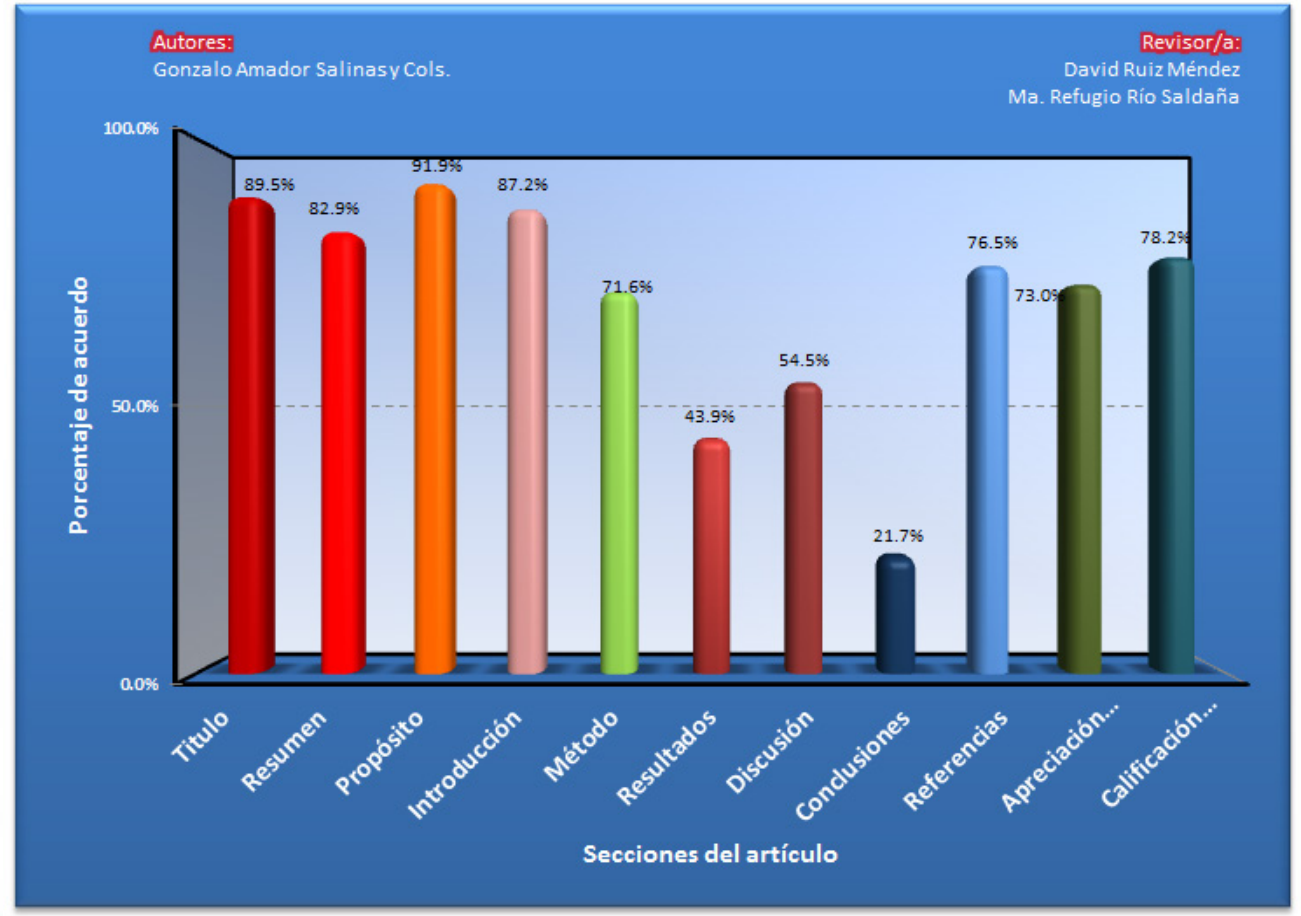




\section{Dimensión Cualitativa}

\begin{tabular}{c|l} 
ReVISOR 1 & \multicolumn{1}{|c}{ ReVISOR 2} \\
\hline David Jiménez Ruíz & $\begin{array}{l}\text { Ma. Refugio Ríos } \\
\text { Saldaña }\end{array}$
\end{tabular}

TÍTULO/AutoríA

El título constituye una parte fundamental del trabajo, puesto que indica al usuario el contenido del mismo de manera clara. En este manuscrito, el título refleja un tema del manuscrito y las variables. Cuestión que no corresponde con las variables involucradas en el estudio. Está redactado utilizando un tono afirmativo. Sin embargo, un aspecto a destacar es que no están referidos los nombres completos de los autores, ya que se utilizan iniciales en algunos de los nombres redactados.

\section{RESUMEN}

La función de un resumen en un artículo científico es permitir al lector identificar de forma clara y precisa los aspectos más básicos de un artículo científico. En este sentido la redacción del mismo debe ser sumamente cuidadosa. En primer término, aunque en el titulo se obtiene una noción muy clara de lo que se pretende en el artículo, cuando se lee el primer párrafo no se especifica la relación entre variables de manera clara, provocando confusión. Solo se menciona que se analizará el efecto de "diferentes variables" las cuales no se mencionan de manera concreta, siendo inferidas en los posteriores párrafos. Tener clara la relación entre variable (dependiente e independiente (s)) es fundamental para que el lector comprenda el propósito del trabajo. Los resultados son mencionados incluyendo el valor $p$ estadístico, que carece de sentido en esta parte del manuscrito ya que no está contextualizado con el valor de la razón $\mathrm{F}$, los grados de libertad del numerador y denominador y la probabilidad exacta de error a. Una consecuencia de no delimitar en principio las variables en las cuales se busca observar relaciones, es que conforme se mencionan los resultados, el lector no tiene claridad al momento comprender los resultados. Se mencionan que hay diferencias estadísticas entre distintas licenciaturas (Odontología, Optometría y Psicología), cuando desde el título y en los primeros párrafos el propósito comparativo entre estos grupos no está mencionado. De este punto se identifica que no hay claridad suficiente en el método a nivel de resumen ya que el lector no sabe que se está haciendo en el estudio, el orden especifico de la aplicación de los instrumentos y su propósito. Por otro lado, la traducción en inglés falla en algunas partes por el pragmatismo de la traducción, la cual promueve sesgos en la traducción de términos. Un ejemplo de esto es la traducción en el resumen de "este trabajo", que en inglés no es "job" ya que esta última refiere a una profesión que se desempeña, la traducción correcta sería "paper" puesto que hacemos referencia al manuscrito. La sintaxis en ingles carece de sentido en algunas partes ya que coincide más con la del español que en la del propio idioma. En algunas partes como en la segunda oración en la segunda coma se omite explícitamente la persona, provocando que la oración se estructure de manera incorrecta. Se sugiere que: - Se redacte de manera clara la relación de variables estudiada en la investigación que reporta el manuscrito, identificando sistemáticamente las variables dependiente e independiente $(\mathrm{s}) \cdot$ Se requiere tener un orden metodológico específico, redactando el propósito de comparación entre grupos (carreras de licenciatura) y la justificación breve de la aplicación de los instrumentos. • Omitir la mención del valor $\mathrm{p}$ estadístico, sin el valor de razón $\mathrm{f}$ y los grados de libertad, ya que su redacción en esta forma carece de significado. - Revisión profunda de la traducción en inglés a nivel de estructura lingüística de la oración, conjugación de estructuras temporales y la correcta traducción de los términos.
Se sugiere identificar todas las palabras clave, así como justificar la autoría múltiple y escribir los nombres y apellidos completos de los autores evitar poner sólo iniciales.

En términos generales el resumen puede considerarse satisfactorio aunque se sugiere atender las sugerencias que se hacen en el texto, especialmente la ortografía, plantear hipótesis, clarificar el objetivo y ajustar el resumen a un máximo de 150 palabras. 


\section{PróPosito del Estudio}

El propósito de una investigación es fundamental puesto que es el eje rector de toda la investigación y del él se desprende la metodología, los resultados y la discusión, que responden a las preguntas de investigación del investigador. Todo esto proceso lleva a aceptar o rechazar la hipótesis de investigación. El propósito del manuscrito fue definido como: "estudiar las diferentes variables como las horas de sueño, patrones de sueño, calidad de sueño, nivel de estudios de las madres, y género en relación al rendimiento escolar". El uso del verbo en el propósito del estudio es crucial. En primer lugar se puede observar una discrepancia inicial, ya que en el titulo se menciona la palabra "efectos" de las horas de sueño sobre el rendimiento escolar y en el propósito del manuscrito, se menciona la palabra estudiar. Estudiar es un término multivoco y amplio que implica la investigación sobre los conceptos mencionados per se. En este sentido, estudiar las variables implicaría, una investigación centrada en cada una de ellas, lo cual escapa del propósito del estudio. En el titulo se aprecia la palabra "efectos", lo cual implica una manipulación directa de las variables independientes sobre la dependiente, lo cual no es congruente con la investigación, puesto que solo se aplicaron los instrumentos para observar la relación entre las variables y de ninguna manera se modificaron las horas de sueño. Por otro lado, en el titulo se menciona claramente la variable de las horas de sueño con el rendimiento escolar, y en el propósito del trabajo se menciona que se pretende observar una relación de variables como horas de suelo, nivel de estudio de la madre y el género de la muestra. No se han definido sistemáticamente las variables. Esta situación se relaciona con la falta de claridad en el resumen. Todo lo anterior provoca que el propósito del estudio no sea claro, que se promueva una confusión sobre lo que se hace y que la metodología no sea totalmente clara ni acorde con la investigación. Por último, se destaca que el propósito del estudio no está redactado en forma de pregunta de investigación o hipótesis. Esto es un problema sumamente grave ya que la hipótesis señala integralmente lo que se pretende demostrar en el estudio, y esta pretensión obedece al valor total del mismo. Cuando el investigador se pregunta ¿Por qué hago mi investigación? La hipótesis define lo que se pretende comprobar y la pregunta que desea contestar. Esta información está relacionada con el valor total del estudio ya que en primer lugar, una investigación se realiza para saber algo, para contestar a una pregunta que tiene el investigador y con la cual pretende contribuir en la ciencia de una disciplina determinada. La carencia explicita de una hipótesis de trabajo implica una fractura grave en el estudio, ya que incluso al hacer un Análisis estadístico para observar la relación entre las variables, se parte de un supuesto para rechazar o aceptar el mismo. La falta de una hipótesis definida puede observarse en estudios exploratorios, pero las características de este estudio no coinciden con un estudio de este tipo Se recomienda: • Revisar de manera sistemática la redacción del propósito del estudio, revisando los verbos utilizados, mencionando las preguntas de investigación y la redacción de la hipótesis. • Definir sistemáticamente las variables involucradas en el estudio con una definición operacional basada en los constructos del marco teórico. - Redactar una hipótesis de trabajo, una hipótesis nula y una alternativa, ya que estas nos permitirán dar un valor explicativo al estudio y definen totalmente el propósito del estudio y el valor científico total del mismo en cuanto a la contribución que se hace a un área de estudio en una disciplina.

Se sugiere que para mejorar la calidad del escrito se establezca la relación del objetivo con los resultados y que los autores se hagan preguntas que puedan contestarse con el análisis de los resultados y propuesta teórica empírica revisada ya que como se presenta hay inconsistencias entre el objetivo y lo que se presenta en los resultados. Además en la sección de método se sugiere describir las características de la muestra encuestada y no en el apartado de resultados. 
Empezando con el propósito de la investigación, como ya antes se señaló, este no es claro, las variables no están completamente definidas de manera operacional, no se definen todas las variables del estudio (la variable de nivel socioeconómico es evaluada pero está totalmente ausente en la redacción del propósito del trabajo) y hay una ausencia de preguntas de investigación y de hipótesis. La ausencia de un planteamiento del problema claro, así como de una hipótesis, hacen que la descripción del campo de estudio pierda significado. La relevancia del tema se define en el manuscrito al citar la importancia que se le da al desempeño de los alumnos de nivel superior y las posibles variables relacionadas con el mismo. No se define claramente la línea de investigación de los autores. El lector puede inferirla de acuerdo a los temas mencionados pero no está explícitamente definida. Otro asunto a destacar es la falta de un modelo teórico general psicológico en el cual se amparen los diferentes estudios citados en el marco teórico. La importancia de contar con una teoría sobre lo psicológico definida obedece a que la definición operacional de las variables es clara y la redacción de las hipótesis es congruente con el propósito de la disciplina. Problema de Investigación. El problema de investigación en el manuscrito no está desarrollado. No hay preguntas de investigación, ni una hipótesis de trabajo con la cual cobre sentido el análisis estadístico para concluir un resultado de investigación. Aunque en el marco teórico se habla claramente de problemas de investigación de otros estudios, en el manuscrito no se identifica un problema de investigación claro. La justificación del estudio, en este sentido, obedece a una ausencia de investigaciones que identifiquen la relación entre las horas de sueño de los estudiantes y su desempeño, aunque en el manuscrito, esta relación no sea definida con precisión y otras variables estén involucradas. Algunos estudios como los de Cladellas et al. (2011) han explorado la relación entre las horas de sueño y el rendimiento escolar, aunque con una muestra con características muy diferentes. Un estudio que pudiera ser de utilidad en el marco teórico. Cladellas, R., Chamarro, A., Badia, M., Oberst, U. y Carbonell, X. (2011) Efectos de las horas y los hábitos de sueño en el rendimiento académico. Cultura y Educación, 23. 1. P. 119-128 Marco Teórico. La elaboración de un marco teórico obedece al planteamiento de un problema y una hipótesis donde se manifieste de manera clara y sistemática la relación entre variables que se pretende observar en el estudio y la definición operacional de cada variable basada en constructo. La conformación del marco teórico en el manuscrito está relacionada con las variables involucradas en la investigación, sin embargo, la falta de claridad del propósito de la investigación, así como la carencia de una hipótesis, hace que el marco teórico no tenga un orden lógico y explicito que debería estar basado en el planteamiento del problema. En este sentido, la función principal del marco teórico en la investigación es clarificar los conceptos involucrados en la definición de una variable. De hecho la variable debería estar definida bajo el constructo, donde su definición operacional estaría claramente definida. La necesidad de una contar con una teoría definida de lo psicológico es fundamental, ya que la relación de las variables y los datos obtenidos en la investigación, tienen sentido solamente bajo la luz de una teoría y de las implicaciones de la misma, de donde se desprenden las hipótesis del investigador. Variables. La definición clara de las variables es fundamental para la pertinencia de la investigación, la metodología escogida y el análisis y la discusión de los datos obtenidos con los instrumentos. Para empezar, se infiriere la relación entre variables independientes y la dependiente, pero esta relación no está explicita en el estudio. Esto puede provocar confusión al momento de identificar el propósito del estudio. Las posibles variables extrañas no se reconocen, ni son mencionadas en el marco teórico. Debe recordarse que la pertinencia de esta definición recae en los tipos de error que puede presentar nuestra investigación donde, una posible consecuencia, podría ser que la relación de variables que registremos no se deba a la variable independiente sino a otra variable.
Al tratarse de

un estudio de tipo empírico, se sugiere considerar las observaciones realizadas en el cuerpo del escrito a través del control de cambios y de los comentarios que ahí se realizan. 


\section{MÉTOdo}

El método de toda investigación es una parte sumamente importante, pues es donde se especifican de manera clara y sistemática las características de la investigación, así como los pasos, instrumentos, muestra, etc. En el manuscrito, en la parte del método hay una división explicita y clara de los participantes, los materiales, instrumentos, procedimientos, etc. Esto brinda luces para identificar partes cruciales del estudio para una posible replicación El diseño del estudio es transversal, lo cual implica que mide las variables en un solo momento temporal. También es un estudio no experimental (no hay muestra aleatoria, grupo (s) control y manipulación directa de la independiente), lo cual es congruente con lo que se hace en el estudio. La investigación reseñada en el manuscrito se ubicaría dentro de un paradigma epistemológico /metodológico cuantitativo, debido a que los instrumentos utilizados para obtener información de las variables definidas son de naturaleza intervalar y por lo tanto asumen continuidad numérica. En este sentido, un instrumento con estas características, permite que los datos obtenidos de los mismos sean susceptibles a un análisis estadístico de varianza como el que se realiza en la investigación (ANOVA). En el apartado de los participantes, se describen sus datos demográficos y se menciona que se realizó un muestreo por conveniencia, lo cual por un lado, no permite generalizar los resultados para una población, pero hace viable una investigación por la facilidad de la obtención de la muestra. En el manuscrito no se menciona de manera clara los procedimientos que aseguran la protección de los participantes de la investigación, como la privacidad de los datos o la existencia de una herramienta como el consentimiento informado. Se recomienda contar con estos apartados. Una cuestión muy importante a señalar es que no hay claridad sobre las variables en las cuales se busca observar una relación. Por un lado, no hay una definición sistemática de las independientes con dependientes, y por lo tanto no hay claridad sobre la hipótesis y el fin del estudio. Por otro lado, en el método se describen datos demográficos, como nivel socioeconómico, género y escolaridad de la madre como variables relacionadas con el rendimiento, relación no especificada de manera clara en el planteamiento del estudio, en resumen y título. No hay hipótesis planteadas. La recomendación en este sentido va dirigida a delimitar de manera sistemática las variables en las cuales se pretende observar una relación y la redacción de una hipótesis que guie la investigación y que defina, en la discusión y conclusiones, el valor explicativo del estudio en la disciplina. De la población y muestra La población objetivo de donde se obtiene la muestra es descrita en el estudio como los estudiantes de nivel superior de las carreras de optometría, psicología y odontología. No se describe a que institución pertenece la muestra obtenida, pudiéndose inferir este dato, pero que, al no estar explicito, puede provocar confusión. Como se mencionó en la parte de arriba, en la investigación se realizó un muestreo por conveniencia. Si bien, este tipo de muestro corresponde a un diseño no experimental, obedece a la conveniencia y facilidad de obtención de la muestra y es generalmente realizado en estudios de tipo exploratorio. Hay congruencia en cuanto al diseño del estudio. Por último, es evidente que la muestra obtenida en el estudio presentaría sesgos por su carácter no aleatorio, por lo que no se podrían realizar generalizaciones en la población objetivo, y la validez del estudio. De los instrumentos En el estudio se especifica la utilización de un cuestionario de perfil socio y el cuestionario de Índice de Calidad de Sueño de Pittsburg (ICSP). En ambos casos se menciona las partes del instrumento y las categorías a evaluar. Debido a que el estudio es de tipo cuantitativo transversal, se debe de contar con las características funcionales de los instrumentos tales como número de ítems, secciones de instrumento y escala de medición. En el caso del ICSP se menciona una consistencia interna, con un coeficiente a de Cronbach de 0.8 , por lo que se asume que la escala de medición es de intervalo. Es importante resaltar que estos datos son pertinentes en la medida que proporcionan al lector los criterios de selección
El escrito puede mejorar si se describen claramente el método y todos sus componentes (características de los participantes, selección de los participantes, las propiedades psicométricas de los instrumentos utilizados, los principios éticos en la investigación (consentimiento informado) y otras observaciones que pueden apreciarse en el texto. 


\section{Resultados}

Resultados Los resultados correspondientes a la investigación reseñada en el manuscrito son los del análisis de varianza (ANOVA). Cuando se reportan los resultados de un análisis de varianza de un factor, además del valor de la razón $F$, deben señalarse los grados de libertad del numerador y denominador (entre paréntesis separados por coma y sin espacio entre el valor $\mathrm{F}$ y el paréntesis), la probabilidad exacta de error $a$, promedios, desviaciones estándar y tamaños muestrales para cada celda (condición) y los resultados del análisis de homogeneidad de las varianzas y del análisis de hipótesis. En los resultados mostrados por el manuscrito únicamente se señala el valor $\mathrm{p}$ indicando diferencias estadísticas, pero, la inclusión de este valor carece de contexto sin el valor de razón Fy el de los grados de libertad. Sin estos datos, se pierde el rigor estadístico que describe los resultados de la varianza y limita el entendimiento de los resultados. Se recomienda apegarse a los criterios de reporte de un análisis de varianza. Una característica fundamental a considerar, es la redacción de los resultados en el manuscrito. La redacción de los resultados debe ser rigurosa y debe de definir claramente las relaciones de las variables observadas con los estadísticos, partiendo de los datos obtenidos en los instrumentos. En la página 8 del manuscrito se presentan una serie de tablas innecesarias, puesto que los datos hay presentados se pueden redactar en prosa. En este sentido, los pies de tabla se encuentran mal redactados. Se recomienda evaluar la pertinencia de las tablas y redactar en prosa los datos señalados en la página 8. Por último, en la página 9 y 10 , se menciona que se comparan las diferencias en las licenciaturas. Esta parte es resaltable debido a que en ninguna parte del método, planteamiento del problema o resumen se dice que se realizarán comparaciones entre grupos. Existe un error metodológico grande, puesto que en ningún momento se definió la pertinencia de hacer comparaciones por estudiantes de carrera. Se recomienda clarificar esta condición en el método.

El escrito puede mejorar si se incluyen en los resultados aquellos que permitan explicar si se logra o no el objetivo y los hallazgos más importantes. Se sugiere que las tablas relativas a las características de los participantes se describan en el método y no en los resultados. Se sugiere también incluir una tabla con los datos de los análisis de anova en todos los casos donde se aplica este estadístico.

\section{DisCusióN}

La discusión en el manuscrito se centra en las variables que se contemplan pero también habla de otras variables que no se especifican en la parte del método y el propósito de la investigación como el turno de los estudiantes o las estrategias de aprendizaje. Se recomienda a los autores, definir con claridad el propósito del estudio, la definición de las variables, la especificación metodológica y el análisis estadístico riguroso para poder realizar una discusión congruente con las variables propuestas.

\section{La discusión puede mejorar se realiza una evaluación de los resultados obtenidos en la investigación y se compara con la de otros hallazgos, se interpretan éstos, se plantean hipótesis alternativas, se discuten las limitaciones de la investigación y se explica si se lograron los objetivos.}

Continúa... 
La parte de las conclusiones es el punto culminante en la investigación. En el manuscrito las interpretaciones se basan en los datos. Sin embargo estas interpretaciones deben de contemplarse en base al estudio y sus características, ya que como se revisó antes, dicha interpretaciones no pueden generalizarse a la población, puesto que no cuentan con la validez necesaria. Se recomienda a los autores tener claro el planteamiento del problema y la hipótesis para concluir en base a estos dos aspectos. Teniendo en cuenta estos factores, se pude identificar y mencionar las contribuciones del manuscrito en la disciplina, lo cual recomendamos. Así mismo, una perspectiva crítica es fundamental, al señalar las limitaciones posibles de la investigación y proponer investigaciones a futuro que pudieran superar dichas limitaciones. Se instiga a concluir bajo esta directriz Por último, es importante señalar las implicaciones teóricas y prácticas del estudio, que obedecen, una vez más, a las contribuciones que se hacen.
Se sugiere mencionar la importancia y aportaciones del estudio al tema que se investiga, así como las repercusiones teóricas y prácticas de los resultados y que faltaría por investigar para darle continuidad al tema que se investiga.

\section{REFERENCIAS}

Se recomienda a los autores revisar los lineamientos del APA para la citación, ya que en algunas partes del texto se encuentran algunos errores como citar entre paréntesis con apellidos e iniciales, la falta de comas y de puntos en las referencias al final del artículo, así como el orden y la forma de estructurar la referencia (volumen, número de páginas, etc.).
El texto, en general, está escrito de una forma correcta. Existen algunos errores de digitación en algunas partes como en la página 2 o en la página 6 . Debido a que no hay un planteamiento claro del problema, por momentos la lectura del trabajo puede confundir al lector. Se recomienda precisión en este aspecto. Se recomienda también la revisión de la citación basada en los criterios del APA.
Se sugiere utilizar principalmente fuentes primarias, actualizadas (los últimos 5 años) y escritas conforme al manual de estilo de la Asociación Psicológica Americana.

Se sugiere que en el escrito se realicen varios ajustes: en cuanto a sistematización, signos de puntuación, reglas de acentuación, actualización de referencias (últimos cinco años) citar fuentes directas y precisar o ampliar los resultados de forma que puedan ser replicables. 
El estudio se puede publicar siempre y cuando se clarifique la metodología, se establezca una hipótesis y en base a estas dos correcciones se desarrolle el manuscrito en todas sus partes.
El tema resulta interesante dado que se investiga una variable que puede afectar el rendimiento académico de los estudiantes, sin embargo, falta claridad en los objetivos, sistematización en el método y procedimiento, descripción clara y detallada de los resultados, discutir los principales hallazgos y contrastarlos con los de estudios previos, revisar fuentes directas y actualizadas. 


\section{IR A LA Historia del Proceso EdITORIAL}


